\title{
Exports and Within-Plant Wage Distributions: \\ Evidence from Mexico
}

\author{
Judith A. Frías \\ David S. Kaplan \\ Eric Verhoogen \\ American Economic Review Papers 83 Proceedings, May 2012 \\ OnLine Appendix
}




\section{Figure A.1. Real exchange rate}

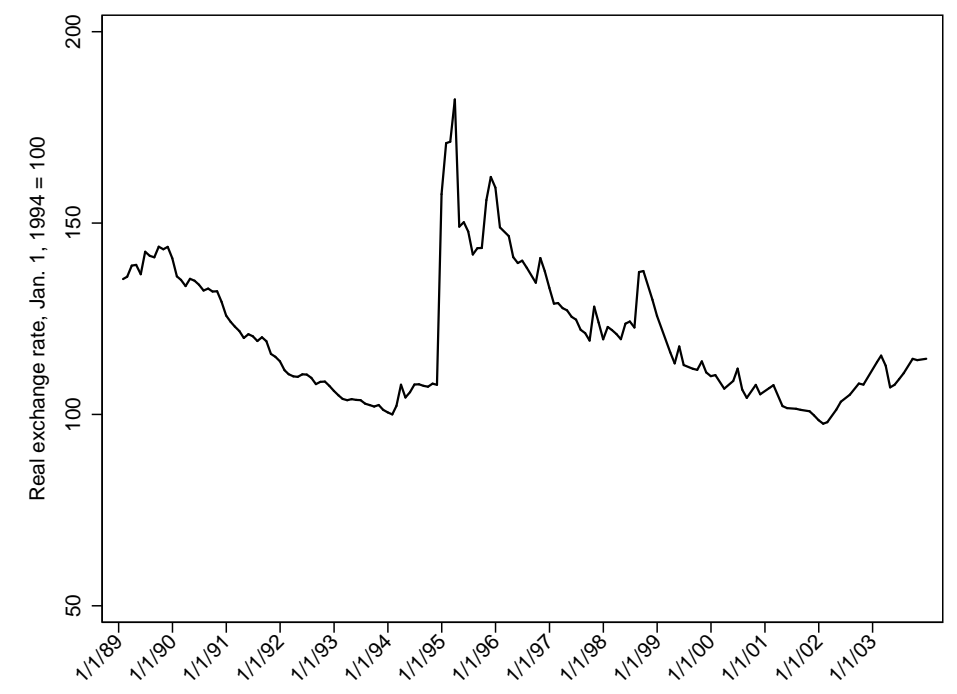

Notes: Real exchange rate calculated as $e \times \mathrm{CPI}(\mathrm{US}) / \mathrm{CPI}(\mathrm{Mexico})$, where $e$ is peso/US\$ nominal exchange rate. Data from IMF International Financial Statistics.

Figure A.2. Shift to export market, EIA panel

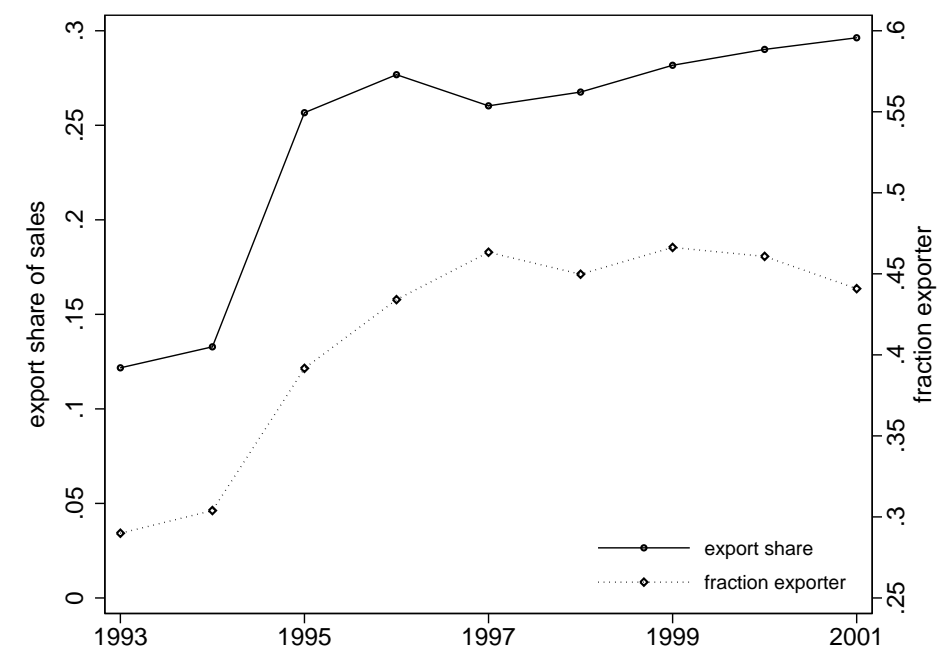

Notes: Data from balanced panel of 2,531 plants for which complete data is available from both EIA and IMSS datasets over the 1993-2003 period. Export percentage of sales calculated as (total exports for all plants)/(total sales for all plants). Plants with exports greater than zero classified as exporters. See text and Frías, Kaplan, and Verhoogen (2011) for further details. 
Table A.1. Summary statistics, plant level, 1993

\begin{tabular}{|c|c|c|c|}
\hline & $\begin{array}{c}\text { non-exporters } \\
(1)\end{array}$ & $\begin{array}{c}\text { exporters } \\
(2)\end{array}$ & $\begin{array}{c}\text { all plants } \\
(3)\end{array}$ \\
\hline \multirow[t]{2}{*}{ Total sales } & 149.12 & 413.91 & 228.21 \\
\hline & $(9.71)$ & $(59.74)$ & $(19.24)$ \\
\hline \multirow[t]{2}{*}{$\mathrm{K} / \mathrm{L}$} & 150.95 & 188.00 & 162.02 \\
\hline & $(10.94)$ & $(14.92)$ & $(8.88)$ \\
\hline \multirow[t]{2}{*}{ Export share of sales } & & 0.15 & 0.04 \\
\hline & & $(0.01)$ & $(0.00)$ \\
\hline \multirow[t]{2}{*}{ Employment (EIA) } & 187.94 & 382.05 & 245.92 \\
\hline & $(6.07)$ & $(27.61)$ & $(9.44)$ \\
\hline \multirow[t]{2}{*}{ Employment (IMSS) } & 178.50 & 386.63 & 240.67 \\
\hline & $(5.74)$ & $(30.81)$ & $(10.22)$ \\
\hline \multirow[t]{2}{*}{ White-collar share of hours (EIA) } & 0.30 & 0.32 & 0.30 \\
\hline & $(0.00)$ & $(0.01)$ & $(0.00)$ \\
\hline \multirow[t]{2}{*}{ Hourly wage (EIA) } & 43.28 & 57.77 & 47.61 \\
\hline & $(0.59)$ & $(1.17)$ & $(0.56)$ \\
\hline \multirow[t]{2}{*}{ White-collar hourly wage (EIA) } & 75.07 & 100.98 & 82.81 \\
\hline & $(1.24)$ & $(2.18)$ & $(1.11)$ \\
\hline \multirow[t]{2}{*}{ Blue-collar hourly wage (EIA) } & 28.87 & 34.86 & 30.66 \\
\hline & $(0.33)$ & $(0.67)$ & $(0.31)$ \\
\hline \multirow[t]{2}{*}{ Avg. daily wage (IMSS) } & 170.42 & 199.57 & 179.12 \\
\hline & $(1.39)$ & $(2.63)$ & $(1.28)$ \\
\hline \multirow{2}{*}{ Median daily wage (IMSS) } & 135.86 & 162.53 & 143.83 \\
\hline & $(1.40)$ & $(2.79)$ & $(1.31)$ \\
\hline $\mathrm{N}$ & 1775 & 756 & 2531 \\
\hline
\end{tabular}

Notes: Table reports statistics using 1993 data from EIA-IMSS 1993-2001 balanced panel. Standard errors of means in parentheses. Exporter defined as export sales $>0$. Export share is fraction of total sales derived from exports. Sales measured in millions of 2002 Mexican pesos, capital-labor ratio in thousands of 2002 pesos, and average daily wage in 2002 pesos. Nominal exchange rate on June 30, 2002: 9.945 pesos/US\$1. EIA variables are reported at plant level. Avg. daily wage (IMSS) calculated by taking average within plant (weighting each worker equally), then taking average across plants (weighting each plant equally). Other IMSS variables calculated at plant level, then simple average taken across plants. For further details, refer to Frías, Kaplan, and Verhoogen (2011). 
Table A.2. Changes in wage inequality indicators, Reduced-form and IV results

\begin{tabular}{|c|c|c|c|c|c|c|c|c|}
\hline & $\begin{array}{c}\triangle \log \\
90 / 10 \\
\text { ratio } \\
(1)\end{array}$ & $\begin{array}{c}\triangle \log \\
75 / 25 \\
\text { ratio } \\
(2)\end{array}$ & $\begin{array}{c}\triangle \log \\
90 / 50 \\
\text { ratio } \\
(3)\end{array}$ & $\begin{array}{c}\triangle \log \\
50 / 10 \\
\text { ratio } \\
(4)\end{array}$ & $\begin{array}{c}\triangle \log \\
75 / 50 \\
\text { ratio } \\
(5)\end{array}$ & $\begin{array}{c}\triangle \log \\
50 / 25 \\
\text { ratio } \\
(6)\end{array}$ & $\begin{array}{c}\triangle \log \\
90 / 75 \\
\text { ratio } \\
(7)\end{array}$ & $\begin{array}{c}\triangle \log \\
25 / 10 \\
\text { ratio } \\
(8)\end{array}$ \\
\hline $\begin{array}{l}\text { A. Reduced form } \\
\text { init. log emp. }{ }^{*} T_{93-97}\end{array}$ & $\begin{array}{c}0.066^{* * *} \\
(0.013)\end{array}$ & $\begin{array}{c}0.035^{* * *} \\
(0.008)\end{array}$ & $\begin{array}{c}0.017 \\
(0.011)\end{array}$ & $\begin{array}{c}0.049^{* * *} \\
(0.009)\end{array}$ & $\begin{array}{l}0.016^{* *} \\
(0.007)\end{array}$ & $\begin{array}{c}0.018^{* * *} \\
(0.005)\end{array}$ & $\begin{array}{c}0.000 \\
(0.009)\end{array}$ & $\begin{array}{c}0.031^{* * *} \\
(0.008)\end{array}$ \\
\hline initial log emp. & $\begin{array}{l}-0.013 \\
(0.009)\end{array}$ & $\begin{array}{l}-0.014^{*} \\
(0.005)\end{array}$ & $\begin{array}{c}0.002 \\
(0.008)\end{array}$ & $\begin{array}{l}-0.015^{*} \\
(0.005)\end{array}$ & $\begin{array}{l}-0.006 \\
(0.004)\end{array}$ & $\begin{array}{l}-0.008 \\
(0.004)\end{array}$ & $\begin{array}{c}0.008 \\
(0.007)\end{array}$ & $\begin{array}{l}-0.008 \\
(0.004)\end{array}$ \\
\hline \multicolumn{9}{|l|}{ B. IV } \\
\hline$\triangle$ export share & $\begin{array}{l}5.390 * * \\
(2.097)\end{array}$ & $\begin{array}{c}2.840^{* *} \\
(1.133)\end{array}$ & $\begin{array}{c}1.367 \\
(1.020)\end{array}$ & $\begin{array}{l}4.023^{* *} \\
(1.587)\end{array}$ & $\begin{array}{l}1.331^{*} \\
(0.695)\end{array}$ & $\begin{array}{c}1.510^{* *} \\
(0.664)\end{array}$ & $\begin{array}{c}0.037 \\
(0.772)\end{array}$ & $\begin{array}{l}2.513^{* *} \\
(1.103)\end{array}$ \\
\hline log emp., initial year & $\begin{array}{l}-0.033 \\
(0.021)\end{array}$ & $\begin{array}{c}-0.024^{* *} \\
(0.011)\end{array}$ & $\begin{array}{l}-0.003 \\
(0.011)\end{array}$ & $\begin{array}{c}-0.030^{* *} \\
(0.015)\end{array}$ & $\begin{array}{l}-0.011 \\
(0.007)\end{array}$ & $\begin{array}{l}-0.013^{*} \\
(0.007)\end{array}$ & $\begin{array}{c}0.008 \\
(0.009)\end{array}$ & $\begin{array}{c}-0.017^{*} \\
(0.010)\end{array}$ \\
\hline industry-year effects & $\mathrm{Y}$ & $\mathrm{Y}$ & $\mathrm{Y}$ & $\mathrm{Y}$ & $\mathrm{Y}$ & $\mathrm{Y}$ & Y & $\mathrm{Y}$ \\
\hline state-year effects & $\mathrm{Y}$ & $\mathrm{Y}$ & $\mathrm{Y}$ & $\mathrm{Y}$ & $\mathrm{Y}$ & $\mathrm{Y}$ & $\mathrm{Y}$ & $\mathrm{Y}$ \\
\hline $\mathrm{N}$ & 5062 & 5062 & 5062 & 5062 & 5062 & 5062 & 5062 & 5062 \\
\hline
\end{tabular}

Notes: Coefficient estimates correspond to differences in estimates from Table 2, Panels C and D in main text; the additional information here is the standard errors. All regressions include 6-digit industry-year and region (state)-year effects. Export share is fraction of total sales derived from exports. Exporter indicator takes the value 1 if export share is greater than zero, and 0 otherwise. Robust standard errors in brackets. ${ }^{*} 10 \%$ level, ${ }^{* *} 5 \%$ level, ${ }^{* * *} 1 \%$ level. 


\section{References}

Frías, J. A., D. S. Kaplan, And E. Verhoogen (2011): "Exports and Wage Premia: Evidence from Mexican Employer-Employee Data," Unpub. paper, Columbia University. 